\title{
AI In Agriculture Using UAV to Detect Weeds
}

\author{
Karanpreet Verma \\ ASET, AMITY University, Noida, Uttar Pradesh (INDIA) \\ Email: karanpvrma@gmail.com \\ Received 18.02.2021, Received in revised form 27.03.2021, Accepted 30.03.2021
}

\begin{abstract}
To keep track of agronomic and environmental variables, UAV's have displayed a tremendous capabilities by capturing pictures at High spatial resolution. Ground-control-points (GCPs) must have to obtain to make sure the precision of the mosaicking process. This study was put through into wheat field naturally overspread by grass leaves and big-leaved at large initial phenological level. UAV flying at altitudes of 30 to $100 \mathrm{~m}$ and using a large number of GCPs (15 to 60), ultra-high spatial resolution ortho-images is able to give rise to ultrahigh spatial resolution ortho-images can be generated with the geo-referencing precision necessary to map small weeds in the field of wheat at very initial phenological stage. 1 acre field was divided into 4 subfields equally, 15 GCP's of different colour configurations were placed in each 4 sub fields providing a precise percentage of weeds in each sub fields detected using UAV. The outcome of the weed detection would give out the exact usage of the pesticide as per the weed detected $(\%)$ in each 4-sub field rather than, spraying the pesticide on the whole entire field.
\end{abstract}

Keywords- Image Processing, Sensor, UAV, Weed, GCPS, Flight Range

\section{INTRODUCTION}

Artificial intelligence refers to the intelligence that machine achieves by the human intelligence that is been programmed in machines so that machines can think, act, speak and perform various tasks. AI keeps on evolving with the innovations, researches,

Inventions and is undergoing a fast transition phase. Agriculture is dominant occupation of India. A remote imagery [2] would help identify land suitable for agricultural activities which was earlier deemed barren. This could help the farmers at times when their lands lose fertility or during times of harsh weather. Location and remote imagery acquisition would help the farmers know what to grow where and when to let it grow its fertility back. AI could help with determining how long the seeds are to kept in their dormant stage to maximise crop produce.

\section{A. Modernized Evolution in Agriculture:}

Strong tillage, monoculture, usage of synthetic fertilizers, technologies associated with irrigation, pest control by chemical methods, genetically manipulated process

The two major variables in using distant imagery for map weeds are resolution of picture spaces and the budburst level of the crop and weeds. The greater and larger is the spatial resolution thus, it would ensure profoundly finer details to distinguish in the image. Moreover, the capacity to distinguish the weeds at an extraordinary phonological level could minimalize the herbicide usage and control associated costs. . It's mandatory to exercise control over weeds at an initial tabula rasa level of the food crop. For instance, when planting the crop and weeds or with two-to-four "true", leaves. The grass weed seedlings detection in the monocotyledonous crops example, wild oats (Avena spp), wild canary grass (Phalaris spp.) in

Winter crop displays much heavier complications than mapping them at a preposterously delayed growth phase under two major reasons:

(i) The spreading of weeds is in little patches, which may show the need to figure with terribly high spatial resolution representational process.

(ii) The Hintergrund of the soil reflective junction with detection consequently an excellent spatial "resolution imagery" maybe, required to spot little weeds and food crop plants at a primitive phenological stage thus on originate the primitive in-season post evolution methods.

The enhancement of UAVs has immense capability to map weeds at variant phenological levels. The studies encircled on three strokes those are: flying altitude, number of GCPs and crop line. Weeds were dispensed in paths within the crops and it displays the spatial-structure which permits mapping, infestations the treatment of herbicides and the infested areas can be set in motion that claims weed presence. The spatial dispersal of weeds crops containing small patches, which describes the implementation and usage of very high spatial resolution imagery. Weed is basically a byproduct of wheat crop that grows in coherence with wheat.

\section{LITERATURE REVIEW}

As discussed in Reference [1], The expert system software applications, which were able to tackle reasoning and solve analysis functions to the depth of the subject to the core at profound levels/stages, 
in which the experts present could solve problems pertaining to farming.

As discussed in Reference [2]. We currently have the most efficient easy sensors fitted to our gadgets and more advanced sensors, including Hyper spectral could boom the range of vegetation kinds that can be

Differentiated and estimate the variety of agricultural parameters and our long-term goal is to apply improved sensing to UAV.

\section{PROPOSED METHODOLOGY}

According to the working implementation of my research paper, the land of 1 acre is divided into 4 sub fields respectively. Consider the following Example, take a square shaped land divided into 4 quadrants. 60 GCP's total allotted. Considering the fact with 4 sub fields, each sub field receive a total of 15 GCP's each. Now Each 15 GCP's per sub field is given a different colored cross on a white square base so as for the drone to identify.

However, the drone will identify from the entire field as though which sub field out of the 4 subfield is sub-field 1 , sub-field 2 , sub-field 3, subfield 4 which is entitled with 15 GCP's per subfield with 4 specific coloured cross mark over a white square base known as GCP. Thus, which quadrant is meant to examine. The GCP's coloured configuration is given below:

Subfield 1-- white square base with neon orange cross mark.

Subfield 2 -- white square base with neon green cross mark.

Subfield 3-- white square base with neon black cross mark.

Subfield 4 -- white square base with neon darkpink cross mark.

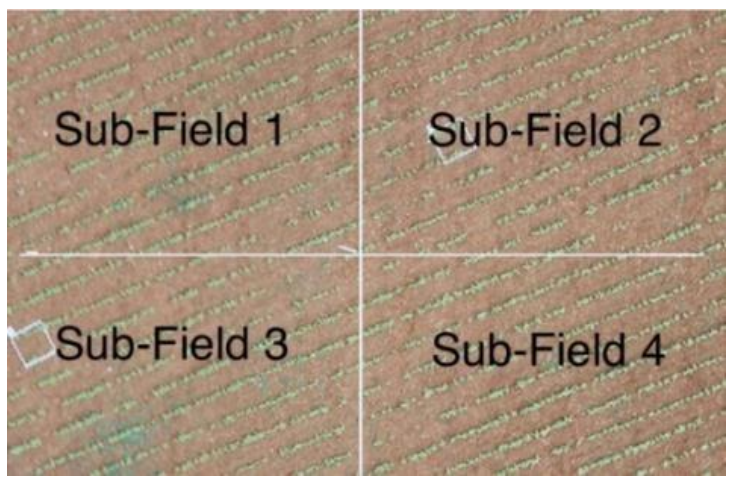

Fig 1: The 1 acre plot that was taken, was divided into 4 sub field.

Key features of denoting each GCP's with a specific colour to the sub field is to help the drone to identify that, as though sub field of the field is to observed and examined (as in figure 4a). In a nutshell, the main motive is that the drone will give the data by image processing, and it'll describe the percentage of weed in that particular sub field from the whole entire owned by the farmer. According for the ease, of the farmers it'll apply $\min / \mathrm{max}$ pesticides in that sub field

Moreover, it'll simply the human labor work where earlier the farmer had to seek and notice each and every edge of the field to locate the weeds. With Artificial intelligence in agriculture, it'll lower the human intervention, bring about greater productivity, enhanced efficiency of work.

\section{A. UAV}

UAV's outlook under the remote sensing process and operations, inclusive of the following: drone's, glider's, (quad-, hexa-, octo-) copter's, helicopter's or chopper with human supervision based on a ground level station (remotely piloted aircraft; RPA) or by the means of any aerial vehicle under coordination.

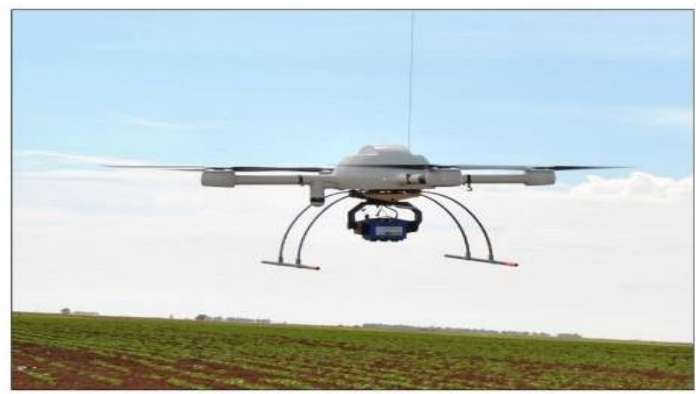

Fig 2: unnamed aerial vehicle (UAV) (7).

UAVs must map read to carry out the remote sensing operation, for the cause it's pre-equipped with variety of sensors and instrument, example the Global- Positioning-Sensors (GPS), Inertial Navigation- Sensors (INS), Micro-ElectroMechanical-Systems (MEMS), gyroscope's and accelerometer's,

Altitude Sensors (AS) or even camera associated sensors among the plenty of them with the multisensory fusion ability and techniques that are required. Video cameras are systems defined in the UAVs for remote sensing. This part of the section pertains to the generic feature, related to visionbased spectrum, onboard UAVs, encircles the spectral visible range, example, the wavelengths varying approximately $390 \mathrm{~nm}$ to $700 \mathrm{~nm}$. Specific video systems are a part of this Applications Section.

\section{B. SENSORS}

Distinction between thermal and infrared sensors because of emitted and cast back energy, respectively. An infrared thermal sensor seize the radiant / gleaming energy, based on the premise that objects with temperatures above an absolute zero emanate infrared transmission as a function of wavelength and temperature. According to the records, ISO 20473, the wavelengths of the 
spectrum bands range: $0.78 \mu \mathrm{m}$ to $3 \mu \mathrm{m}$ (nearinfrared), $3 \mu \mathrm{m}$ to $50 \mu \mathrm{m}$ (middle- infrared), and 50 $\mu \mathrm{m}$ to $1000 \mu \mathrm{m}$ (far-infrared). Some among the following, spectral ranges can be combined into multispectral or Hyper-spectral sensors altogether with visible range of spectrum. Infrared and thermal cameras are devices inclined of operating in unfavorable weather conditions or low radiation, monitoring during the night.

Multispectral Sensors (MS) basically are nonscanning, and they, generally, layout lower image resolutions in comparison to hyper-spectral sensors. The CCD rooted camera with four channels (blue: $420 \mathrm{~nm}-520 \mathrm{~nm}$; green: $520 \mathrm{~nm}-600 \mathrm{~nm}$; red: 630 nm-690 nm; NIR: $760 \mathrm{~nm}-900 \mathrm{~nm}$ ) was scaled onboard an UAV aimed on the ground surface. Multispectral and hyper-spectral sensors are usually used jointly with other sensors with greater imprinted high performance to enhance the remote sensing ability of the UAV.

The coordinates of each and every edge of the exploratory and examinary fields have been gathered with GPS to prepare the flight course. This imagery was gathered with two distinct cameras set up separately in a quadcopter UAV, model md4- 1000 along with the base station (in figure 2) encircled with four brushless motors also a CFD upgraded propellers. Operating at a speed of $12 \mathrm{~m} / \mathrm{s}$, and ascend at a rate of $7.5 \mathrm{~m} / \mathrm{s}$. This UAV lengthen of $1.3 \mathrm{~m}$ and a utmost height in which it can take-off weight of $6 \mathrm{~kg}$ with the Recommended payload capacity is $800 \mathrm{~g}$, although, threshold payload capacity is $1.2 \mathrm{~kg}$. Range can be expanded up to $40 \mathrm{~km}$ via GPS waypoint navigation, and flight tolerance is 88 minutes.

The base station, brought about by microdrones, uses md-cockpit application software to eye track and exercise control on the flights.

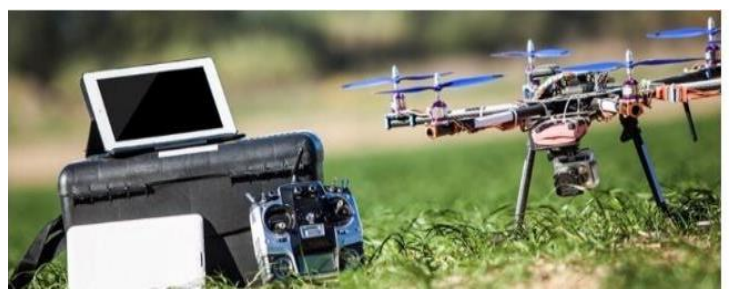

Fig 3: md4 1000 micro drone with its base station the UAV.

Imagery accounted at two distinctive altitudes: 30 and $100 \mathrm{~m}$. spectrum camera, model Olympus PEN E- PM1.Which had 1.3-megapixel images. This camera has a B(450nm), G(530nm), R(670 and 700nm), R edge(740nm) and near-infrared (NIR, $780 \mathrm{~nm}$ ) spectral region band pass of $10-\mathrm{nm}$ full width at half limit and middle wavelengths. Comprehensive details on UAV

Flight setup and concise information on the basis of aircraft and the cameras [7]. The images captured with the visible spectra camera were utilized straight after it downloaded to the computer, on the other hand those cumulated them it, the six images taken at each waypoint in a single file-[7]. The Pixel Wrench 2 software endowed with the multispectral camera it performed the alignment process.

\section{LOCALY AND DISTANT IMAGERY PROCUREMENT}

In accordance to respective analysis, testified in wheat crops locale in the Khanna province in North Punjab (India). One plot of roughly 1.00 acre was taken and later it was divided into 4 subplots as in figure 3. The geographia attainment coordinates (Universal- Transverse-Mercator-System, zone 30 North, WGS-84) Of the extreme most were at X = $33341 \mathrm{~m}, \mathrm{Y}=844656 \mathrm{~m}$ respectively. Wheat crops were planted in pre-September 2019 in rows which were $10 \mathrm{~cm}$ spreaded by (Malva spp. and Sinapisspp.) and wild canary grass. The crops and weeds were at the initial development of vegetative level (4-5 true leaves). At the study intriguing the plot, the pictures were taken by the Micro-drone MD4-1000 quad-rotor UAV fitted with the base station (figure 2) and an Olympus EP-1 camera (red, green and blue bands) on the pre-February 2019. The main agenda of planning contained the recording of the co-ordinates for the flight region in every field followed by the next step, that is to transmit the information/data from the UAV using memory card to the PC, in order to ground station generate flight rows, flight height range. All over the entire area, a totality of 60 Artificial GCPs (A white square target with 4 specific coloured cross sign, Fig. 3) a white square frame was put down for weed sampling next to every GCP under latter weed discrimination (Fig. 4).

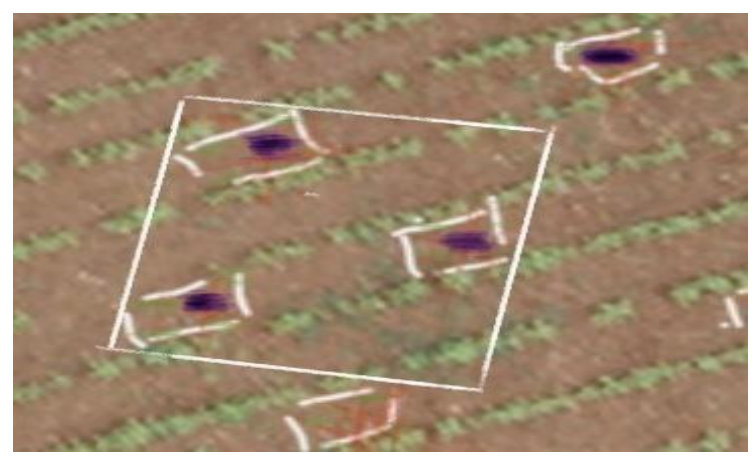

Fig 4: Out of total 60 GCP's, 15 GCP's per sub field allocated with a different colour configurations, to give out information about weeds to UAV (7)

\section{IMAGE PROCESSING}

Images nonheritable with UAVs cowl comparatively tiny land areas; thence, automatic mosaicking and rectification are needed for covering larger areas once required. Mosaicking and orthorectification are product obtained in period of time from video streams. Image process techniques were used to eliminate foggy images and pictures with 
excessive overlapping the attention track visual spectra and its exposure strokes of the UAV, pertaining the angles

These frameworks were utilized as an input data to the Leica Photogrammetric Suite 2010, software under the ortho-rectification by the "aerotriangulation and mosaicking". Camera calibration components were testified and calculated by juxtapose overlapping (9) dimension of one after the other images throughout the aero- triangulation Precision Agric process. Thereafter, the images have been confounded into the seamless ortho-mosaicked picture of each and every corner of the wheat field.

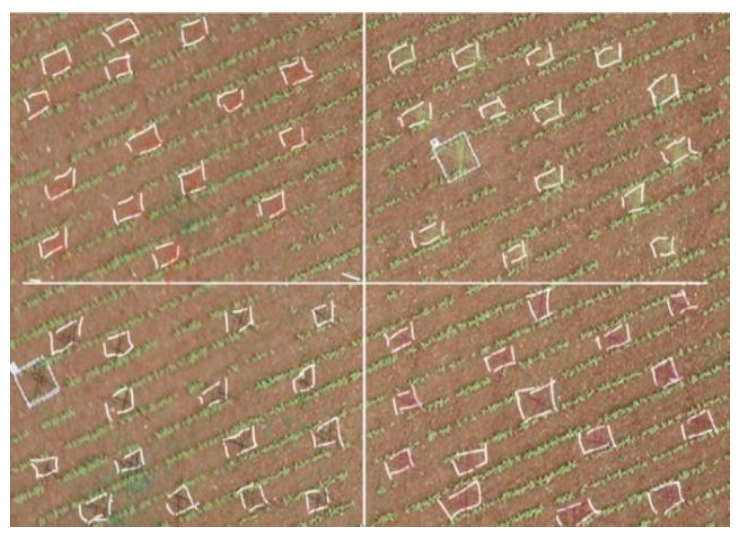

Fig 5: Detail of the file created, in one of the 49 square frames for wheat crop (green) and weed (violet) classes (7).

\section{E. FLIGHT RANGE}

A series of 37,120 photos correlative with $30,100 \mathrm{~m}$ flying altitudes, severally, were taken in thought and refined into action for orthorectification and mosaicking exploitation Leica Photogrammetry Suite (LPS) package to showcase a particular ortho-image of each section of the wheat field. LPS allocates coordinates to the image that's taken because the input, 60-GCPs placed. The amount of GCPs used for fabricating every mosaick was equivalent to the single image count of each array of series (e.g. for a 100m flight height, no below 15 GCPs are often used )

Table 1, showcases the weeds that were detected in the various 4 sub fields in (\%) using UAV, flying at the altitude $30 \mathrm{~m}$ on observation day 1 and then gaped 15 days with an observation

\begin{tabular}{cccc}
\multicolumn{3}{c}{ day 2.} & \\
SUB-FIELDS & WEED DETECT USING UAV & IMAGES & PIXEL \\
& AT FLYING ALTITUDE 30M & CLICKED & SIZE(M) \\
& & & \\
OBSER. DAY-1 OBSER.-DAY-2 & & \\
\hline
\end{tabular}

\begin{tabular}{lcccc}
\hline Sub- Field 1. & $15 \%$ & $28 \%$ & 76 & 0.0127 \\
Sub- Field 2. & $26 \%$ & $52 \%$ & 119 & 0.0164 \\
Sub- Field 3. & $5 \%$ & $11 \%$ & 37 & 0.0112 \\
Sub- Field 4. & $12 \%$ & $34 \%$ & 83 & 0.0131
\end{tabular}

\section{RESULTS}

The number of pictures and also the flight extent lengthwise required to hide the whole entire analysis examined has increased from 40 to 120 images and from 10 to $30 \mathrm{~min}$ at $30 \mathrm{~m}$ altitude.

A decrement within the flying altitude strippeddown the world coated by every of the subsequent single image, that finally showcases associate degree sweetening in each the sequence of series of pictures and also the complexness and enormity of the image mosaicking series of steps to amass an ortho-image casing the complete space.

Observation, by applying UAV at $30 \mathrm{~m}$ altitude on the observation day 1 , the weeds present in various 4 sub fields were less in percentage, as mentioned above in the table 1 . As, after 15 days on the observation day 2 , it was observed that the weed present in the various 4 sub fields were slightly increased from the previous observation day. Thus, required a large number of images that were captured by the UAV and stored in the CF cards in the UAV, later on transferred to the computer into the software so that images could be processed into the software to detect accurate no. of percentage of weeds in the 4 various sub fields on both the observation days. So, that weeds could be detected accurately and more efficiently moreover, for each sub field the percentage of weeds examined can be proved to be fruitful.

All photograph arrayed series were clutched at each of the locations that is, close mid noon over the 2 chosen respective days gaped with 15 days so as to examine it. Weather conditions were more or less alike in both of the picture series. The handiest great distinction changed into that, with the associated weather conditions at the 1 st observation day on the field plot have been moderately cloudy while the pictures had been taken, after 15 days, it was sunny sufficient on the commentary day 2 . The only prime distinction that holds significance below repeatable course of study, in which imagery had to be obtained for the 'same sites at variant times.

The concluding spatial resolutions of the orthomosaicks were 0.74 and $2.47 \mathrm{~cm}$ for flight elevation of 30 and $100 \mathrm{~m}$ respectively. The outcome didn't showcase greater differences in precision between the period of time of the altitude examined (30-100 $\mathrm{m})$. Therefore, with an enhancement in the wide variety of GCPs would extraordinarily upgrade the geo-referencing precision.

\section{CONCLUSION}

Flight altitude is a crucial tool parameter to require into thought whereas accounting remote pictures victimization the UAV. as a result of the abstraction formation of uneven segregation of weeds permits mapping of overrun and A-infested zones/sections, the most motive were to look at and apprize the 
Grass patches at previous phenological steps victimization UAV imaging and to form a time framed and economical and effective weed examining and program supported site-specific chemical treatments in keeping with weed cowl or weed layers .However, there have been no greatly georeferencing issues within the ortho-mosaics designed by UAV bagged with RGB or MultiSpectral(MS)cameras flying at thirty and a hundred $\mathrm{m}$ altitudes[9]. Moreover, the quantity of pictures required to hide the entire field at thirty $m$ altitude with the visible-spectra camera was abundant less than for the Multi-Spectral (MS) camera, showcasing it as a limiting issue because of ability of UAV energy constraints [12].

At a low height, victimization the visible camera, the UAV clicked mostly finer minute abstraction resolution imaging than at an equivalent altitude victimization the assistance of a multi-spectral camera. By for the case, whereas selecting the proper flight altitude, 2 strokes are additionally crucial than image falsification : the primary one is that the threshold abstraction resolution needed to tell apart between weeds and crops and also the other is the variety of single pictures required to drill the complete space, as a bigger variety of single pictures build the ortho-rectifying and mosaicking tougher processes, additional thus, a really bigger variety of pictures per acre greatly increments at very low altitudes transportation concerning associate straight line curve.

The operation time period is additionally a matter of concern that's subjected to restrict by the battery efficiency of UAV. These variables have nice implementation for weed mapping in previous season that, in accordance to the result, involved 2 crucial confined conditions :1) to offer out remote pictures with a rather fine abstraction resolution to substantiate weed segregation. 2) to least the inoperation time and also the variety of pictures to minimize the constraint of flight fundamental quantity and image mosaicking wholly.

Considering the above analysis made, where a field is divided into 4 sub-fields, in which the 15 GCP's are allotted to each and every 4 subfields. The series of steps involved to take more images by the UAV in the field on the particular observation days, tend to give more accuracy to detect weed percentage in every sub fields. For instance, rather than taking the entire field only a sub part of the field would be taken and could easily draft following essential points:

1) The number of pesticides that was ought to be applied not over the whole field, but only on that sub field where the percentage of weed is more. The utilization of pesticides would be confined to a certain subfield depending on the weed percentage. So, by this pesticide's usage would lower down.

2)Where the percentage of weed is maximum out of the 4 subfields, detected by the UAV the farmer will directly go to that particular sub field to spray pesticides over it.

\section{FUTURE WORK}

The future scope under the model proposed, can be adding a special feature or a mechanism in the UAV so as to inform the farmer about the exact amount of pesticide required for each and every sub field and also the whole entire field. For example, UAV detected in a sub section $10 \%$ of weeds and in accordance to that $20 \%$ in amount is the pesticides required. Further which, the technique of speech recognition can be induced in the model for the ease of the farmers under the agriculture to expand the application of AI under agriculture.

\section{ACKNOWLEDGMENT}

I'm sincerely thankful to Amity University, Noida for providing me with the opportunity to write a research paper on the topic "AI IN AGRICULTURE UAB TO DETECT WEEDS".

I'm utterly grateful and thankful to the authors under the reference section so as to serve as an ignition to make the paper meaningful and interesting.

I'm also thankful to the librarian and the computer lab in charge of Amity University, Noida who have helped me during the course of this research paper in different ways.

Through the research paper I have learnt a lot about the perspicacity of artificial intelligence. It has helped greatly for my analysis of the research paper.

\section{REFERENCES}

[1] McKinion, J. M., and H. E. Lemmon. "Expert systems for agriculture." Computers and Electronics in Agriculture 1.1 (1985): pg. no. 31-40

[2] Gay, Alan P., et al. "Developing unmanned aerial vehicles for local and flexible environmental and agricultural monitoring." Proceedings of RSPSoc 2009 Annual Conference. Vol. 8. No. 11. 2009.

[3] Raafat, Amany I., Mona Eid, and Magda B. El- Arnaouty. "Radiation synthesis of superabsorbent CMC based hydrogels for agriculture applications." Nuclear Instruments and Methods in Physics Research Section B: Beam Interactions with Materials and Atoms 283 (2012): 71-76.

[4] Stockwell, V. O., and B. Duffy. "Use of antibiotics in plant agriculture." Revue Scientifique Et Technique-Office International Des Epizooties 31.1 (2012).

[5] Peña, José Manuel, et al. "Weed mapping in early- season maize fields using object-based analysis of unmanned aerial vehicle (UAV) images." PloS one 8.10 (2013).

[6] Huang, Yanbo, et al. "Development and prospect of unmanned aerial vehicle technologies for agricultural production management." International Journal of Agricultural and Biological Engineering 6.3 (2013): pg. no. $1-10$.

[7] Torres-Sánchez, Jorge, et al. "Multi-temporal mapping of the vegetation fraction in early-season wheat fields using images from UAV." Computers and Electronics in Agriculture 103 (2014): ph. No. 104- 113. 